Revista General de Información y Documentación ISSN: 1132-1873

https://dx.doi.org/10.5209/rgid.70069

\title{
Las redes sociales como herramienta educativa a través de la producción científica
}

\author{
Gonzalo Lorenzo Lledó ${ }^{1}$
}

Recibido: 25 de abril de 2020 / Aceptado: 4 de junio de 2020

\begin{abstract}
Resumen. Las sociedades actuales han ido evolucionando hacía nuevas formas de comunicación que ha proporcionado la tecnología. Las redes sociales han permitido la creación de entornos interactivos para compartir información y crear conocimiento. La influencia de estas herramientas en los diferentes contextos han lugar a un incremento en las investigaciones sobre su uso en el campo educativo. Por ello, el objetivo de esta investigación ha sido analizar la producción científica sobre el uso de las redes sociales como herramientas formativas durante periodo 2009-2018. Para ello se han utilizado diversas bases de datos entre las que destacan la Science Citation Index, Emerging Source Citation Index y la Science Citation Index Expanded. Se introdujeron una serie de palabras clave que permitieron obtener una muestra de 252 documentos que fueron analizados en función de una serie de indicadores bibliométricos como el índice de inmediatez, el índice de aislamiento o el número de publicaciones anuales. Los resultados constatan que el periodo de mayor producción ha sido el comprendido entre 2015-2018, siendo la gran mayoría de los artículos publicados en revistas del Q1 tanto en la Social Science Citation Index y la Science Citation Index Expanded. Por otro lado, existe un $19 \%$ de solape entre las revistas de ambas bases de datos. Asimismo, los equipos de trabajo oscilan entre uno y tres autores. Como futuras líneas de trabajo se plantea completar la muestra de documentos desde una perspectiva más de análisis de temáticas.
\end{abstract}

Palabras clave: redes sociales; ISI web of Knowledge; sociedad de la información.

\section{[en] Social networks as an educational tool through scientific production}

\begin{abstract}
Today's societies have been evolving towards new forms of communication provided by technology. Social networks have enabled the creation of interactive environments for sharing information and creating knowledge. The influence of these tools in different contexts is being recognized in the scientific literature. Therefore, the objective of this research has been to analyze the scientific production on the use of social networks as training tools during the period 2009-2018. For this purpose, several databases have been used, including the Science Citation Index, Emerging Source Citation Index and Science Citation Index Expanded. A series of key words were introduced that allowed us to obtain a sample of 252 documents that were analyzed according to a series of bibliometric indicators such as the immediacy index, the isolation index or the number of annual publications. The results show that the period of greatest production has been between 2015-2018,
\end{abstract}

1 Universidad de Alicante. Departamento de Psicología Evolutiva y Didáctica.

E-mail: glledo@ua.es 
with the vast majority of articles published in Q1 journals in both the Social Science Citation Index and the Science Citation Index Expanded. On the other hand, there is a 19\% overlap between the journals in both databases. Likewise, the working teams range from one to three authors. As future lines of work, the idea is to complete the sample of documents from a more thematic analysis perspective.

Keywords: social networks; ISI web of Knowledge; information society.

Sumario. 1. Introducción. 2. Método. 3. Resultados. 4. Discusiones. 5. Conclusiones. 6. Referencias bibliográficas.

Cómo citar Lorenzo Lledó, G. (2020) Las redes sociales como herramienta educativa a través de la producción científica, en Revista General de Información y Documentación 30 (1), 243-260.

\section{Introducción}

La tecnología es uno de los pilares que generan crecimiento y desarrollo en las sociedades actuales. Existe una gran cantidad de actividades que se realizan en la vida diaria que utilizan la tecnología como herramienta conductora y de transmisión. Cada uno de nosotros queremos compartir y enviar documentos en los diferentes formatos existentes (imagen, vídeo...).

Como consecuencia se ha creado un ecosistema formado por las diversas herramientas de internet que van a permitir crear y modificar contenidos al usuario y trabajar de forma colaborativa es lo que O’Reilly (2005) acuñó con el termino Web 2.0. Para O’Reilly (2005) la Web 2.0 permite al usuario ser un creador de contenidos en el entorno Web recién creado. De la misma manera para Martín, Hernández-Suárez \& Mendoza-Lizcano (2017), el nuevo termino Web 2.0 se refiere al paso de entornos Web más estáticos a entornos más dinámicos. Estos permiten la incorporación de aplicaciones que están orientadas al servicio del usuario con una función comunicativa y trabajando las comunidades de usuarios. Asimismo, Cabada, Estrada, Sanchez, Sandoval, Velazquez, \& Barrientos, J. (2009) establece una primera clasificación sobre las herramientas de la Web 2.0: wikis, redes sociales, servicios de marcadores sociales, mensajería instantánea y audio-vídeo (YouTube). Posteriormente, García-Martín \& García-Sánchez (2013), establecen dos grupos principales de aplicaciones que constituyen la Web 2.0. Uno de ellos está formado por las aplicaciones emocionales. La finalidad de estas herramientas es fomentar la relación personal entre los diferentes usuarios mediante la creación de perfiles o la publicación de contenidos multimedia. Ejemplos de ellas serían Facebook, Instagram, Twitter. Mientras que el segundo grupo de herramientas se focalizan en aplicaciones instrumentales. Ejemplos de este segundo tipo son las Wikis, Blogs, Google Docs.

A partir del análisis de las diferentes herramientas de la Web 2.0, es importante enfatizar según apuntan Hemmi, Bayne \& Land (2009); Kaplan \& Haenline (2010); Gruzd, Staves \& Wilk (2011) que el termino Web 2.0 también puede ser utilizado para una de las utilidades que tienen un mayor peso y que son las redes sociales y medios sociales. Ampliando la definición del término medios sociales o "social media”, Joosten (2012) afirma que los medios sociales son los diferentes 
sistemas tecnológicos cuyo objetivo es promover la colaboración y comunicación entre los diversos usuarios. En línea con lo indicado anteriormente Kwon \& Yixing (2010) expone que las redes sociales han sido concebidas como espacios web personales que tienen como objetivo la construcción de relaciones interpersonales mediante la acción de compartir información con cualquier tipo de usuario sea conocido o no. Para Ajjan \& Hartshorne (2008); Robbie, \& Zeeng (2008), las redes sociales han cambiado la forma en la que unas personas acceden a otras, además de las formas de interacción y finalmente se ha visto influido por la forma en la que se comparte y se transmite la información. El tipo de información que se puede compartir serán fotos, videos o enlaces a determinados contenidos (Malesky \& Peters, 2012). Estas nuevas formas de comunicación permiten escribir mensajes online en el perfil de otro usuario (Begovic, 2011) además de establecer nuevas amistades (Teclehaimanot \& Hickman, 2009).

Tomando como referencia las definiciones y funcionalidades que presentan las redes sociales, Castro (2019) establece que las redes sociales más utilizadas son Facebook, Twitter, Facebook, Instagram, WhatsApp, Twitter, Youtube y LinkedIn. Asimismo, García, Froment, Bohórquez \& Vieira (2017) concluyen que estas herramientas tienen como publico principal los adolescentes y el alumnado universitario. A pesar de ello, es tal la presencia de los medios sociales que en los últimos años la población adulta también ha sido asidua en estos medios. Aunque utilizado con una finalidad informativa más que para compartir información. Por otro lado, estas nuevas herramientas para Eid \& Al-Jabari (2016), generan la creación de espacios para discutir temas o conceptos y colaborar para completar tareas o proyectos escolares. Las redes sociales proporcionan formas convenientes y rápidas de intercambio de conocimiento y colaboración entre pares que puede ser el método preferido de aprendizaje. Para Eid \& Al-Jabari (2016) estas herramientas fomentan el autoaprendizaje de los alumnos y fomentan un cambio en el esquema tradicional formativo. Además de eliminar las limitaciones de tiempo, espacio y lugar es lo que se conoce como aprendizaje ubicuo (Burbules, 2014). Asimismo Ko (2012) expone como estas herramientas han generado un incremento de la motivación en el aprendizaje de las personas. En la misma línea Lin \& Tasai (2011) afirman que las redes sociales proporcionan un mejor entorno de aprendizaje mediante la interacción constante y un aprendizaje a más alto nivel entre los estudiantes.

Como consecuencia de ello, las redes sociales permiten ampliar y abrir fronteras a la formación evitando que se quede recluida a las aulas. En esta línea, Latchem (2018) expone que la formación no está limitada al contexto educativo formal que engloba desde Infantil hasta la universidad sino que existe otra parte no formal. Este tipo de formación abarca cualquier actividad educativa que tiene como objetivo proporcionar determinados tipos de aprendizaje a grupos concretos de población como pueden ser la gente mayor o los niños. Además y según Hager (2012), los conocimientos y aptitudes que acumulan las personas a partir de las experiencias vividas van a completar esa educación no formal que recibimos.

Desde la justificación expuesta, el presente estudio tiene como objetivo realizar un análisis sobre el estado de la producción científica sobre la utilización de las 
redes sociales como herramientas educativas. Como consecuencia de ello, se han establecido una serie de indicadores para analizar la documentación durante el periodo 2009-2018. A partir del objetivo planteado se han establecido una serie de preguntas de investigación a las que se pretende dar respuesta en este estudio.

- ¿Cuál ha sido el periodo de mayor producción científica y el número de artículos?

- ¿Un elevado número de citas lleva asociado un elevado valor del índice de inmediatez?

- ¿ ¿Según el análisis de la vida media de las citas el tópico de estudio es muy dinámico?

- ¿Los papers en revistas representan el tipo de documento más abundante en la muestra?

- ¿Cuáles son las categorías de la Web of Science que trabajan la temática de estudio?

- ¿Cuáles son las áreas de la Web of Science más trabajadas?

- ¿Los países más productores tienen índices de aislamiento elevados?

- ¿Los autores más productores forman parte de los centros de mayor producción?

- ¿ ¿Los equipos de trabajo están constituidos por más de 5 autores?

- ¿La gran mayoría de las revistas analizadas forman parte del Q1 del Social Citation

- Index y del Science Citation Expanded?

- ¿Las actas de congresos tienen una mayor representación que los libros en las colecciones de libros?

\section{Método}

La investigación llevaba a cabo se sustenta en el diseño bibliométrico descriptivoretrospectivo que según Montero \& León (2007) permite el análisis y la clasificación de los documentos. Para la implementación de esta metodología de trabajo se deben llevar a cabo una serie de pasos para su correcto desarrollo: Para comenzar es necesario realizar el proceso de búsqueda y selección de la información más adecuada. Posteriormente se deben establecer una serie de descriptores que permitan la clasificación y finalmente el análisis de datos (Rosa, Huertas \& Blanco, 1996).

\subsection{Muestra}

La muestra está constituida por 256 documentos publicados durante el periodo 2009-2018. Se ha escogido este periodo ya que antes de 2009 no existen publicaciones sobre la temática y se excluyó el año 2019 porque aún no ha finalizado. Los diversos documentos analizados estaban incluidos en las diferentes 
bases de datos que a continuación indicamos: Science Citation Index Expanded, Social Science Citation Index, Arts \& Humanities Citation Index, Conference Proceedings Citation Index Science, Conference Proceedings Citation Index-Social Science \& Humanities, Book Citation Index Science, Book Citation Index-Social Science \& Humanities, Current Chemical Reactions, Index Chemicus, Emerging Source Citation Index. A todas estas bases de datos se tiene acceso desde el portal de la ISI web of Science. Asimismo, la primera fase de búsqueda y selección de la información se realizó en el mes de enero de 2019, mientras que el proceso de lectura y análisis se llevó a cabo en febrero-marzo 2019.

\subsection{Diseño y procedimiento}

Con el objetivo de poder abarcar un mayor número de artículos sobre la temática de las redes sociales como elemento formativo, en la pestaña principal de la Web of Science se introdujeron los siguientes descriptores de búsqueda

TOPIC: (("Social Network" or "Redes Sociales"OR "Facebook" OR "Twitter" OR "Instagram" OR "Youtube" OR "Whatsapp"OR "Linkedin") AND ("Education" or "Higher Education")) AND TITLE: (("Social Netwoork" or "Redes Sociales"OR "Facebook" OR "Twitter" OR "Instagram" OR "Youtube" OR "Whatsapp"OR "Linkedin") AND ("Education" or "Higher Education"))

Se escogieron los términos Social Network y Redes Sociales porque son los más generales utilizados para definir comunidades de usuarios (online o no) que tiene una serie de intereses en común y que intercambian información (García, Froment, Bohórquez \& Vieira, 2017). No se escogió el termino Social Media en la búsqueda ya que trabaja más el ámbito de la comunicación (Joosten, 2012). Por otro lado, se escogieron las redes sociales Facebook, Twitter, Instagram, Youtube, WhatsApp y LinkedIn, que opinión de Castro (2019) son las más populares entre los adolescentes y universitarios. En esta línea, investigaciones como las de Brenley \& Coven (2018) y Awidi, Paynter \& Vujosevic (2019) afirman que Facebook, Twitter y Youtube son las redes sociales más utilizadas por los usuarios para compartir información, de ahí que su introducción en la docencia sea entendida como un complemento (Ruiz, 2019). Asimismo, Instagram, Linkedin o Whatsapp son consideradas como un elemento vital en la creación de ese perfil digital de relación entre profesores y estudiantes. Algunas redes sociales que están teniendo una gran expansión como Tik Tok (Xu, Yang \& Zhang (2019), no fuera utilizada como descriptor en el estudio. Las razones fueron según Xu, et al. (2019) que esta red tiene como objetivo la difusión de los contenidos en el ámbito de la comunicación no siendo la Educación uno de sus campos principales de trabajo. Otras de las razones por las cuales no fue incluida como descriptor son las dificultades para la gestión de la calidad de los contenidos y la abundancia de contenidos fake (falsos).

Finalmente se escogió el término Education para englobar todas las actividades formativas formales y no formales en los niveles no superiores además del aspecto 
formativo de las redes sociales. Asimismo, el termino Higher Education puede englobar todas las actividades realizadas en los niveles superiores de educación.

La aplicación de las líneas de código anteriores nos permitió obtener una muestra de 272 documentos. Para obtener una mayor rigurosidad en los resultados se decidió excluir el año 2019, ya que aún no ha finalizado y por tanto se pueden incorporar nuevos documentos que restarían validez a la investigación realizada. A partir de la muestra obtenida se llevaron a cabo las siguientes fases que constituyeron el protocolo de trabajo de la investigación.

- Análisis de la información asociada a los documentos. En este sentido es importante tener en cuenta el título del documento, el año de publicación, su ámbito y la autoría junto con la institución de procedencia. Por otro lado, no se deben olvidar las características de la revista como por ejemplo el título, temática, cuartil además del idioma y el país de referencia

- Construcción de los indicadores de la ciencia bibliométrica. Entre los que destacan el índice de inmediatez, índice de aislamiento, calidad de las revistas, índice de colaboración y el índice de productividad.

En la figura 1 se presenta el diseño y procedimiento de la investigación:

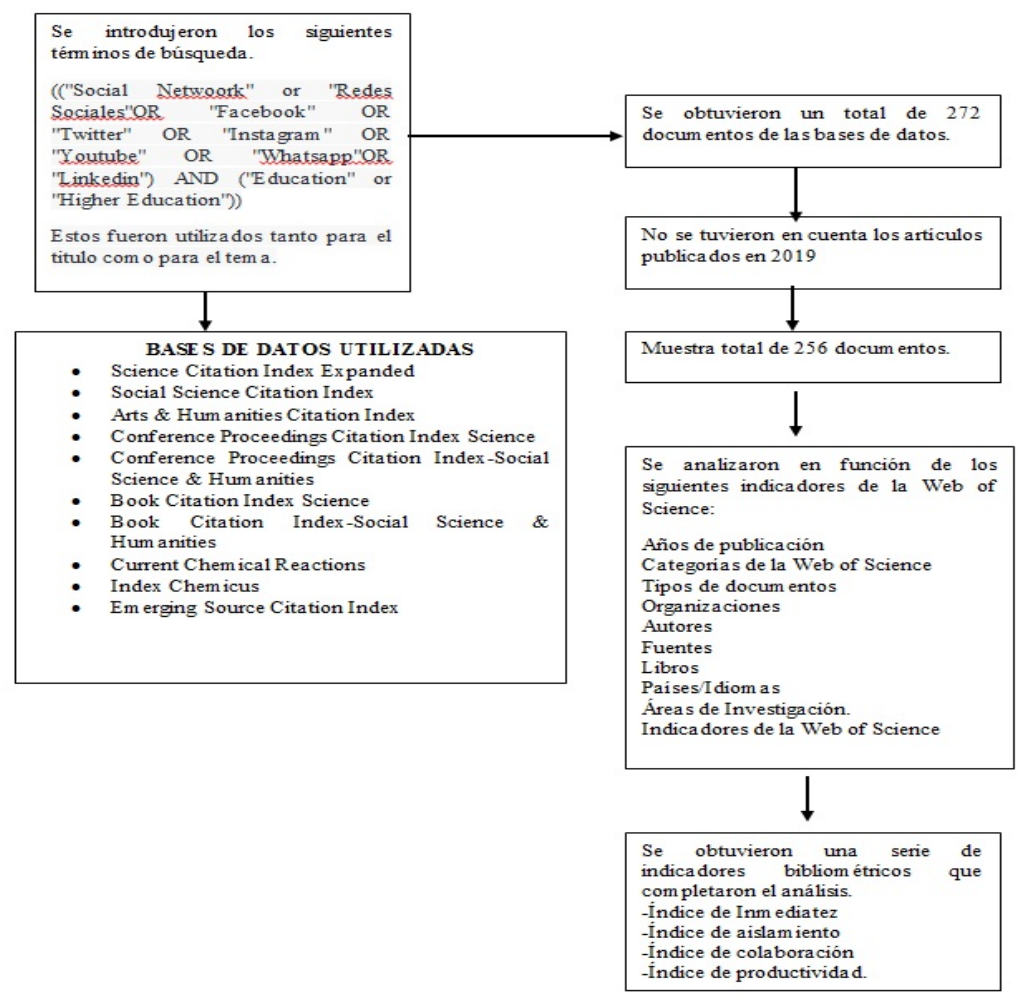

Figura 1. Procedimiento y diseño de la investigación 


\section{Resultados}

Se presentan a continuación los resultados obtenidos a partir de las variables del estudio en función de las preguntas de investigación planteadas.

\subsection{Número de artículos publicados}

La primera de las variables utilizadas ha sido el número de artículos publicados. Como se puede observar en la figura 2, existen dos periodos de producción claramente diferenciados 2009-2013 y 2015-2018 donde se acumulan más del 70\% de los documentos. En el año 2014 se presenta una ligera disminución de la producción.

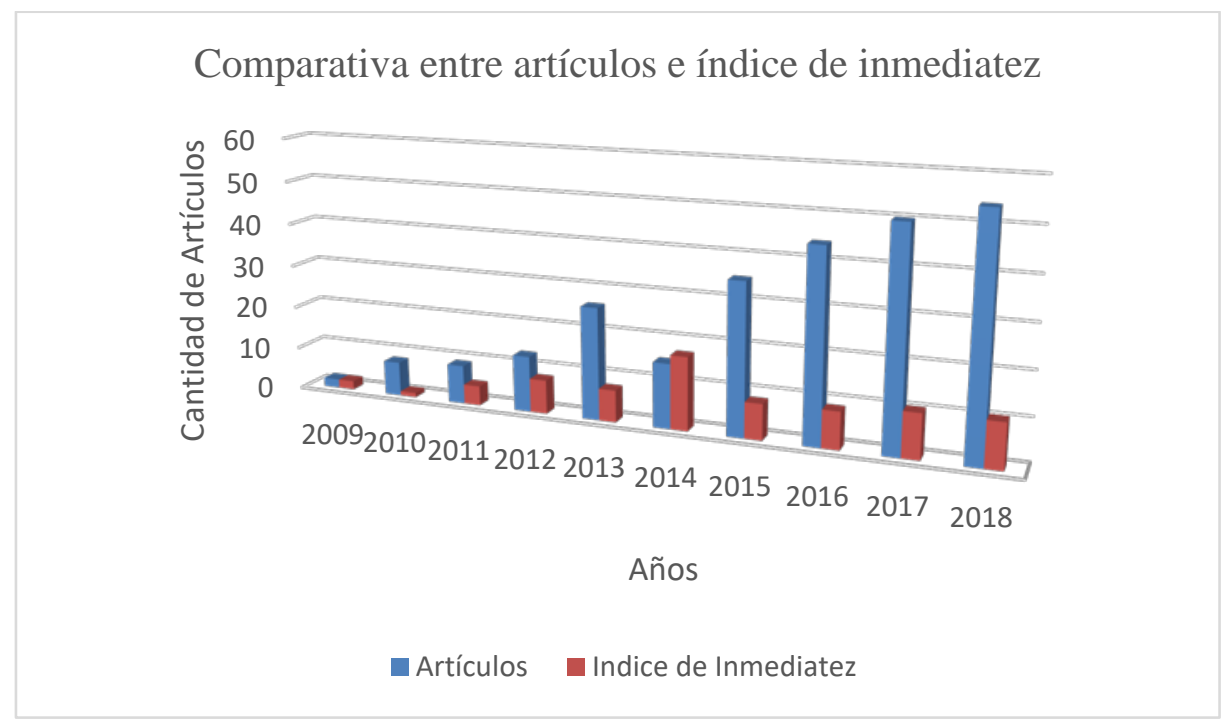

Figura 2. Comparativa entre artículos e índice de inmediatez

\subsection{Número de citas e índice de inmediatez}

El número de citas es otra de las herramientas utilizadas en la investigación planteada. Durante el periodo analizado es a partir del año 2012 cuando se va produciendo un incremento en el número de citas pasando en el 2011 de 41 citas a 102 citas en el 2012 un incremento del 125\%. A partir de ese año se produce un crecimiento constante hasta el año 2018 donde alcanza un tope de 570 citas. A partir del estudio de la evolución del número de citas es necesario mostrar aquellos artículos que han recibido el mayor número de citas anuales. Esta información queda recogida en la tabla 1. El artículo de Roblyer, McDaniel, Webb, Herman \& Witty (2010) recibe un total de 442 citas, seguido del artículo de Selwyn (2009) con 356 citas. 
Tabla 1. Los 10 artículos más citados según la web of Science en el uso de las redes sociales

\begin{tabular}{l}
\hline Referencia \\
\hline Roblyer, M., McDaniel, M., Webb, M., Herman, J., \& \\
Witty, J. (2010). Findings on Facebook in higher \\
Education: A comparison of college faculty and students \\
uses and perceptions of social networking sites. Internet
\end{tabular}
and Higher Education, 13(3), 134-140

Selwyn, N. (2009). Faceworking: exploring student's 356 Education related use of Facebook. Learning Media and Technology, 34(2), 157-174.

Kabilan, M., Ahmad, N., Abidin, M., (2010). Facebook: An online environment for learning of English in institution of Higher Education. Internet and Higher Education, 13(4), 179-187

Veletsianos, G. (2012). Higher Education scholar's participation and practices on Twitter. Journal of Computer Assisted Learning, 28(4), 336-349.

Kassens-Noor, E. (2012). Twitter as a teaching practice to Número de citas 442 enhace active and informal learning in higher Education: The case of sustanaible tweets. Active Learning in Higher Education, 13(1), 9-21

Attal, D., Cowher, M., Al-Hamadani, M., Schoger, J., 82 Staley, A., \& Landercasper, J. (2015). Twitter Social Media is a effective tool for Breast Cancer Patient Education and Suport: Patient-Reported Outcomes by Survey. Journal of Medical Internet Research, 17(7), 188.

Jaffar, A. (2012). Youtube: An Emerging tool in anatomy Education. Anatomical Sciences Education, 5(3), 158-164

Forgie, S., Duff, J., \& Shelley, R. (2013). Twelve tips for 68 using Twitter as a learning tool in Medical Educational. Medical Teacher, 35(1), 8-14

Stefania, M., \& Ranieri, M. (2016). Facebook and the 57 others. Potentials and obstacles of Social Media for teaching in higher Education. Computers \& Education, 95(1), 216-230

Lohse, B. (2013). Facebook is an effective strategy to recruit low-income women to online Nutrition Education. Journal of Nutrition Education and Behavior, 45(1), 6976 
El análisis del número de citas se complementa con el estudio del índice de inmediatez (Rueda-Clausen, VillaRoel \& Rueda-Clausen, 2005), que nos indica la frecuencia de citación de los artículos. El estudio de la figura 2 nos da a entender que no existe un crecimiento constante. El índice va creciendo marcando un máximo en 2017 con un valor de 17, el año siguiente se produce un descenso hasta el valor 8.56, volviendo al crecimiento constante hasta los 10.56 del 2018. El estudio de la evolución del número de citas debe ser completado con el análisis de la vida media de las citas según lo indicado por Burton \& Kebler (1960). Para ello se realiza el cálculo de la mediana de la distribución del número de citas, que nos dio un valor de 8.09 .

\subsection{Tipos de documentos}

La muestra de estudio esta compuesta por un 54\% de artículos de revista, 25\% de Proceedings de congresos, un $9 \%$ de meeting abstracts, un 5\% de otros documentos, y un 7\% de capítulos de libros.

\subsection{Categorías de la Web of Science}

Cada documento que se publica en las bases de datos de la Web of Science es clasificada según el área de la materia publicada. Para los resultados presentados se obtuvieron las siguientes categorías Education Educational Research con el 44\% de los documentos, Education Science Disciplines con el 9\% de los materiales y el tercer lugar lo ocupa la categoría Communication con el 6\%.

\section{5. Áreas de investigación}

Cada una de las categorías de las Web of Science, esta asignada a un área de investigación. Bajo este criterio, el 52\% de los documentos estan incluidos dentro del área Education Educational Research, Computer Science tiene el 11\% de la documentación recogida. Finalmente el área de Communication presenta el 6\%.

\subsection{Países e idiomas}

Los países con mayor número de literatura científica sobre la temática de las redes sociales son USA e Inglaterra que entre ambos ocupan el 39\% de la producción científica. Asimismo, el inglés es el idioma predominante con el 92\% de la producción, el castellano ocupa el segundo lugar con el $4 \%$, seguido del portugués con el 3\%. Con el objetivo de analizar la difusión que tiene la producción científica de un determinado país se ha utilizado el índice de aislamiento (López-Piñero y Terrada, 1992). USA e Inglaterra tienen los índices de aislamiento más elevados con un $46 \%$ y un $20 \%$ respectivamente. Por el contrario Australia y España solo tienen índices del 2\%. 


\subsection{Autores}

Para poder obtener una imagen mas realista del tópico de estudio se hace necesario analizar cuáles son los autores mas productivos y su centro de procedencia. Veletsianos, de la Universidad de Texas Austin tiene el mayor índice de productividad (Lotka, 1926) con 0.70. Por el contrario no es de las más productivas, el primer lugar le corresponde a la Johns Hopkins University con el $3 \%$ de la producción. El segundo de los autores más productivos es Kimmons con un índice de 0.60 perteneciente a la Brighman Young University, que tampoco se encuentra entre las más productivas. Esta posición la ocupa la University of California System con un 3\% de la producción. La tercera posición de los autores más productivos es para para Attal (2015) con un índice de 0.48 y pertenece a la University of California Los Ángeles. Este centro ocupa la misma posición en cuento a los centros más productores con el 3\% de la producción. Finalmente la figura 3 nos indica la distribución de las publicaciones según el número de autores. El análisis nos indica que más del $60 \%$ de los documentos han sido realizados por equipos de trabajo que oscilan entre 1-3 autores. Para obtener una mayor rigurosidad científica del hallazgo obtenido se ha calculado el índice de colaboración (Ferreiro \& Martín, 1997) con un valor de 2.79 .

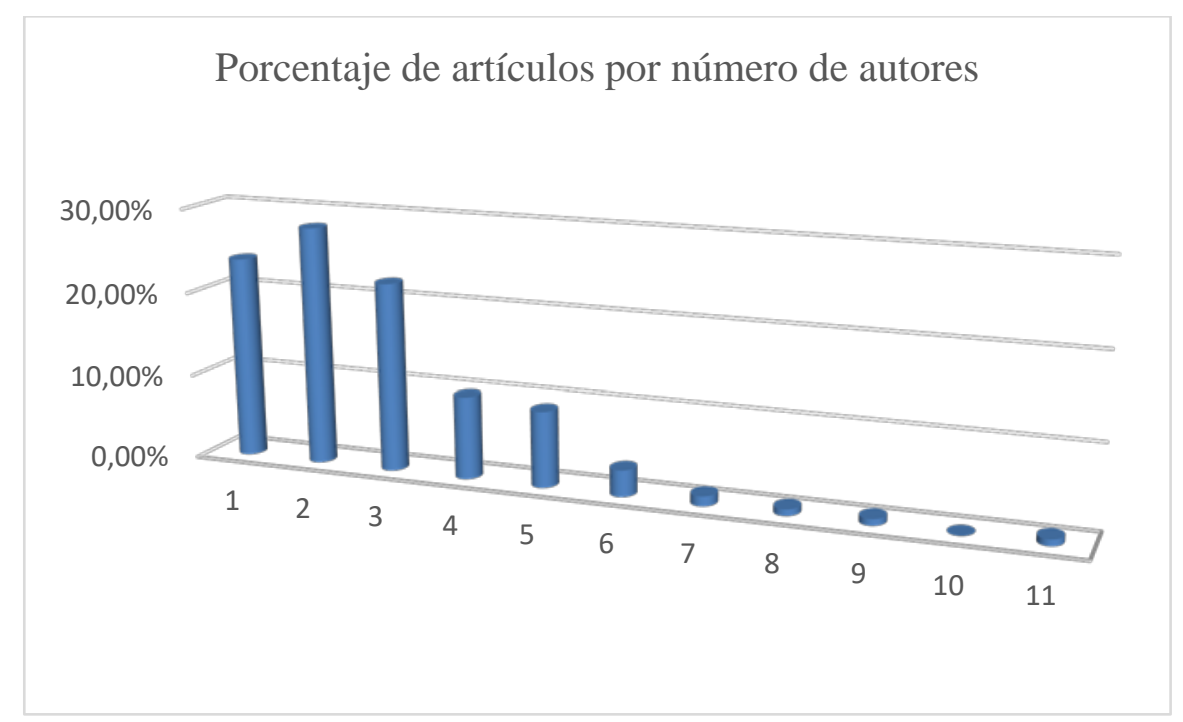

Figura 3. Porcentaje de artículos por número de autores.

\subsection{Calidad de las revistas por bases de datos}

El análisis de la calidad de las revistas se va a realizar en función a las bases de datos existentes en el Web of Science. De un total de 163 revistas indexadas, el 29\% pertenecen a la Emerging Source Citation Index el 33\% a la Social Science Citation Index mientras que el 38\% forman parte del Science Citation Index Expanded. Entre estas dos bases de datos existe un solape del 22\% de sus revistas. 
Para el Emerging Source Citation Index, las tres revistas mas productivas son: Journal of Marketing of Higher Education, Education and Information Technologies y EduWeb Revista te Tecnología de la Información y Comunicación en Educación, teniendo una producción de 3, 2, 2 artículos respectivamente. Siendo Education and Information Technologies la que presenta un promedio de citación por artículo más elevada con 5.5. Esta misma revista es la que presente un índice de autocitación más elevado con el 9\%.

En cuanto a la Social Science Citation Index, la figura 4 indica que el 46\% de las revistas son del Q1 donde se publican el 55\% de los artículos. Existe un 26\% de revistas del Q2 que acumulan el 30\% de los artículos. Finalmente el 17\% de las revistas son del Q3 y se producen el 12\% de los artículos.

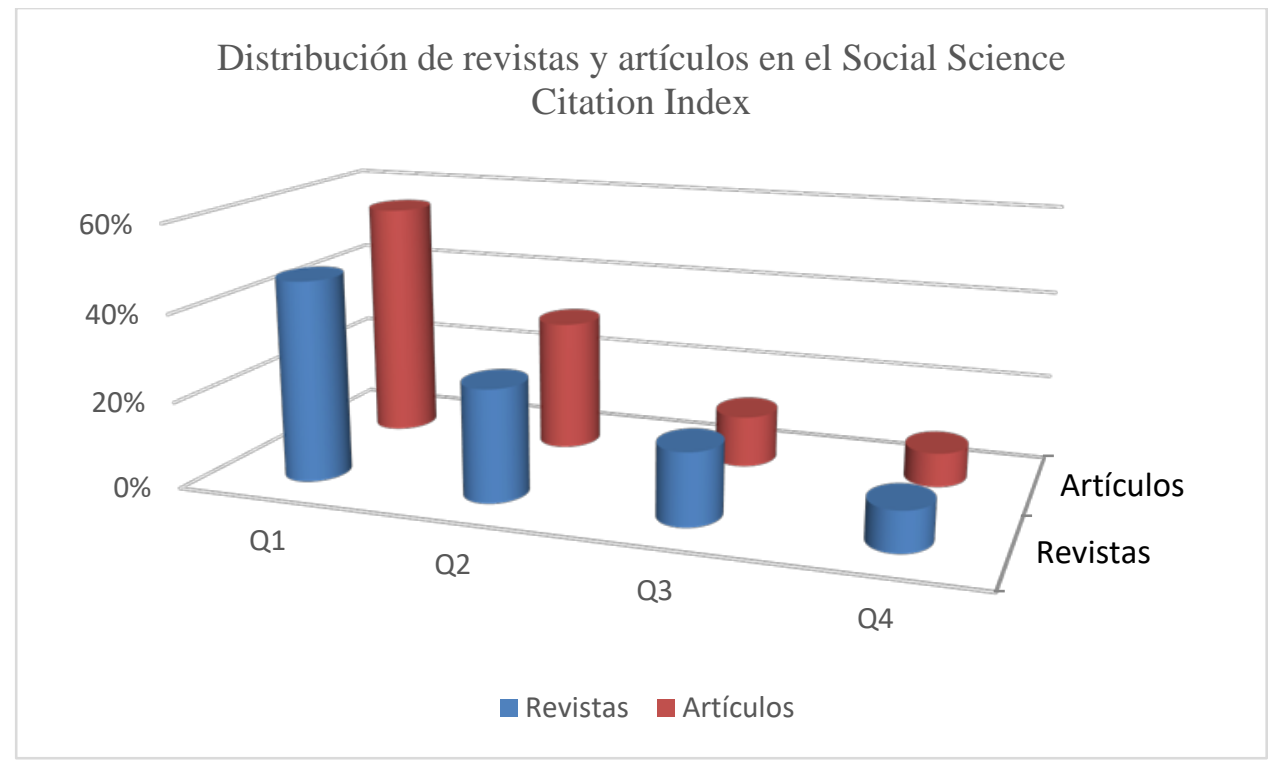

Figura 4. Calidad de las revistas y artículos en la Social Science Citation Index.

Dentro de esta base, la revista Anatomical Science Education acumula el 5\% de los artículos con un 3.28\% de autocitación (Vallejo-Ruiz 2005) y un promedio de 45.75 citas por artículo. Seguida de Computers \& Education con el $4 \%$ de la producción, un $0 \%$ de autocitación y un promedio de 40.33 citas por artículo. Mientras que el tercer lugar lo ocupa la revista International Review of Research in Open and Distributed Learning con el 4\% de la producción, un 0\% de autocitación y un promedio de 2 citas por artículo.

En cuanto a la Science Citation Index Expanded, la figura 5 indica que el 50\% de las revistas están indexadas en el Q1 generando el 62\% de los artículos, el 16\% son revistas del Q2 con el 12\% de los artículos mientras que el 14\% son revistas del Q3 o Q4 con el 11\% de los artículos. 


\section{Distribución de artículos y revistas en el Science Citation Index Expanded}

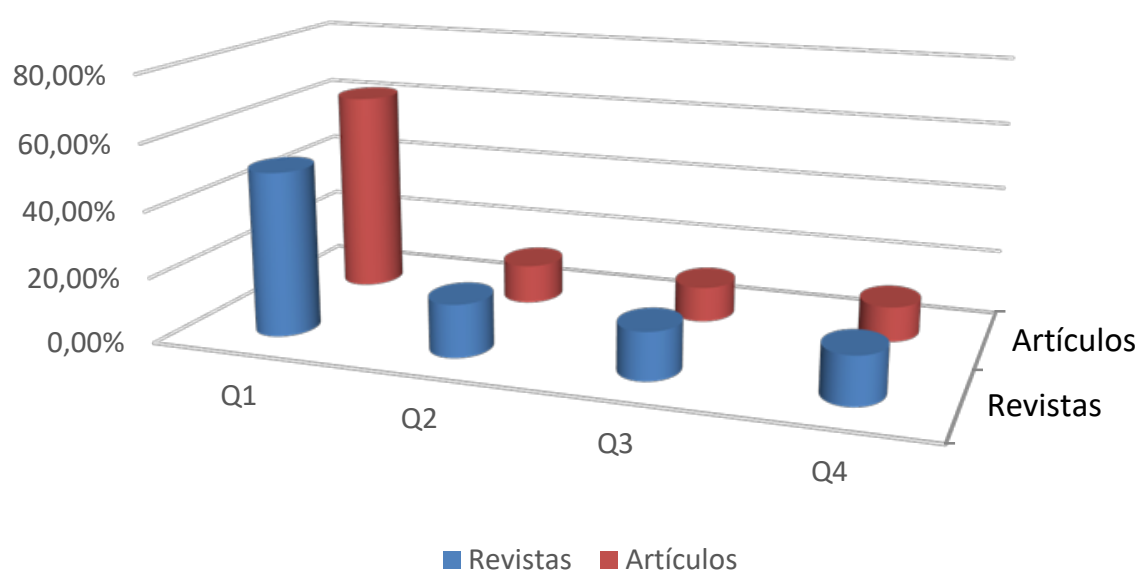

Figura 5. Calidad de las revistas y artículos en la Science Citation Index Expanded

La revista con mayor producción es la Anatomical Science Education que genera el 7\% de los documentos, tiene un 5.95\% de autocitación y el promedio de citas por artículo es de 30.83. Seguida de la revista Aesthetic Surgery Journal con un $5 \%$ de la documentación, presenta un $22.22 \%$ de autocitación y un promedio de citas por artículo de 4.5. Para finalizar la revista Computers \& Education con el 4\% de la producción, un $0 \%$ de autocitación y un promedio de 40.33 citas por artículo

\subsection{Libros y congresos}

El análisis de estas variables se realizó conjuntamente ya que muchos congresos posteriormente han sido editados como libros. De las 30 colecciones existentes, el $53 \%$ correspondieron a libros, mientras que el $47 \%$ hacen referencia a las actas de congresos. De los 56 artículos, el 29\% (16 artículos) han sido publicados en Edulearn Proceedings y en Inted Proceedings. La tercera posición la ocupa el libro titulado "Cutting Edge Technologies in Higher Education" indexado en el Book Citation Index y que acumula el 7\% de la producción. El segundo libro en importancia es el titulado "Educating Educators with Social Media".

\section{Discusiones}

En el presente estudio sobre el estado del arte de la producción científica de la utilización de las redes sociales como herramienta educativa ha sido estructurado en función de una serie de indicadores bibliométricos definidos previamente.

En cuanto a los dos primeros indicadores en los que se basa el estudio son el número de artículos y el número de citas, ambos se relacionan mediante el índice de 
inmediatez. El periodo de estudio abarcó el período comprendido entre 2009-2018. Los años anteriores al periodo analizado los estudios son prácticamente inexistentes ya que las principales redes sociales, excepto Instagram creada en el 2010, vieron la luz en el periodo 2002-2009. La primera de ellas LinkedIn en 2002 y la última Whatsapp en 2009. Se ha constatado como el periodo de estudio se puede dividir en dos subperíodos el intervalo 2009-2013 y el 2015-2018. Tanto en el primero intervalo como en el segundo existe un crecimiento constante debido a que como indica Roblyer et al. (2010), las redes sociales se han convertido en una herramienta que ha traspasado todos los ámbitos de la vida diaria y se ha convertido en un recurso muy valioso que sirve como elemento de apoyo en las comunicaciones formativas y colaborativo para las tareas desarrolladas con la institución. Por otro lado en el año 2018 se alcanza un mayor número de citas con 570. A pesar de ello, el índice de Inmediatez más elevado tiene lugar en el 2014 con una puntuación de 17.00. Según lo establecido por Rueda-Clausen, VillaRoel \& Rueda-Clausen (2005) este valor tan elevado se debe a que durante ese año se produce una menor producción que en los anteriores y existe un número elevado de citas, como se consecuencia la relación resultante es elevada con respecto a las anteriores y posteriores. Finalmente existe un valor medio de las citas de 8.09, según Burton-Kebler (1960) esto nos indica que los documentos que han recibido las citas tienen 8 años de antigüedad. Por tanto y según el periodo estudiado se esta realizando una renovación de la literatura científica.

El siguiente indicador analizado ha sido los documentos. En la muestra de estudio el 55\% de los documentos fueron revistas mientras que el $25 \%$ fueron parte de los Proceedings a congresos. A diferencia de otras investigaciones como la realizada por Lorenzo \& Scagliarini (2018), en este estudio también se tuvieron en cuenta los libros que constituyeron el 7\% de los documentos. A pesar de analizar un período relativamente corto, el elevado número de artículos en revistas nos indica que estamos ante un campo bastante consolidado.

Otro de los indicadores importantes ha sido las categorías de la Web of Science y las áreas de investigación de la WOS. En cuanto a las categorías de la Web of Science, Education Educational Research con el $44 \%$ de los documentos y Education Scientific Disciplines con el 9\% son las más representativas y Communication ocupa el tercer lugar con el 6\%. Esta última posición esta justificada según lo indicado por Joosten (2012) que enfatiza en el papel comunicador de las redes sociales. La primera posición de Education Educational Research es consecuencia de lo indicado por Eid \& Al-Jabari (2016) que indica que estas herramientas son espacios formativos y colaborativos de aprendizaje para desarrollar y construir nuevos conceptos. La categoría Education Scientific Disciplines abarca ámbitos médicos, educativos y psicológicos, por este motivo cuatro de los diez documentos con mayor visibilidad son de esta categoría. En cuanto a las áreas de investigación de la Web of Science, Education Educational Research ocupa el primer lugar con el 52\%, seguido de Computer Science con el $11 \%$. Esto es consecuencia de lo indicado por García-Martín \& García-Sánchez (2013) que afirman que las redes sociales son aplicaciones que permiten la creación de entornos virtuales online donde se producen interacciones entre usuarios. Por tanto existe un gran componente tecnológico. 
Otro de los indicadores que ha sido utilizado ha sido los países productores/Idioma y el índice de aislamiento López-Piñero \& Terrada, (1992). Tanto Estados Unidos como Inglaterra son los países más productores sobre la temática con un total del 39\% de la producción científica siendo el Inglés el idioma predominante. Por el contrario son estos países los que presenten un índice de aislamiento mas elevado con valores del $46 \%$ y del $20 \%$ respectivamente. Esto según López-Piñero \& Terrada, (1992) nos da a entender que los autores de dichos países están utilizando un porcentaje de investigaciones internacionales menor del 85\%. En cambio países como España y Australia presentan valores del 2\% de índice de aislamiento, lo que indica que su producción científica esta siendo exportada el extranjero.

Los autores y las instituciones de procedencia son otros de los indicadores analizados. Casi el $75 \%$ de la literatura científica esta producida por equipos que oscilan entre uno y tres autores. Según Ferreiro \& Martín (1997) para obtener una mayor rigurosidad científica es necesario calcular el índice de colaboración que nos dio un valor de 2.7. Los equipos de 4 o más autores solo constituyen el $25 \%$ de la muestra. Este dato según Heffner (1981) nos indica que las investigaciones reciben poca ayuda financiera y por tanto un mayor número de participantes no implica que existe un mayor aporte monetario a la investigación. Del mismo modo y según lo indicado anteriormente, solo Attai que tiene un índice de productividad (Lotka, 1926) de 0.48 pertenece al tercer centro productor que es la Universidad California los Ángeles. Tanto Veletsianos como Kimmons con valores de productividad de 0.70 y 0.60 respectivamente no pertenecen a los centros más productivos. Mientras que los centros de mayor productividad están situados en los países mas productores como son Estados Unidos e Inglaterra.

En cuanto a la calidad de las revistas, se ha podido observar como es un ámbito bastante interdisciplinar ya que el $38 \%$ pertenecen al Science Citation Index Expanded (SCIE), y el 33\% al Social Science Citation Index (SSCI), existiendo un 19\% de solape entre ambas bases de datos. De todas formas se ha podido observar que en ambos casos las revistas del Q1 son mayoritarias alcanzando valores del $46 \%$ y del 50\% respectivamente además todas tienen como procedencia los países más productores (Estados Unidos e Inglaterra). La publicación mayoritaria en revistas del Q1 da lugar a que mas del 50\% de los artículos estén aglutinados en ellas. Siendo las revistas del Q4 una presencia marginal con el 9\% en el Social Science Citation Index. Por otro tanto en la Emerging Source Citation Index como en el SCIE y SSCI, las revistas con mayor producción alcanzan valores que oscilan entre el 5\%-7\%. Dándose el caso que la revista mas productora en el SSIE y el SSCI es Anatomical Science Education y además mostrando de esta forma el solape entre ambas bases y la diversidad de enfoques del campo. Resulta significativo indicar que la segunda revista más productora de la SCIE presente un índice de autocitación (Camps,2008) del 23\%. Esto puede significar la existencia de autores muy productivos que publiquen en esta revista y citen sus propios trabajos.

Para finalizar el último de los indicadores que ha sido analizado son los libros y congresos. A pesar de que solo el $47 \%$ de los libros proceden de congresos, casi el 
$30 \%$ de los documentos generados proceden de las colecciones de dos congresos EDULEARN e INTED. Esto se debe a que existe una gran proliferación de libros pero de poca visibilidad en cuanto al número de citas mientras que existen tres congresos entre los mas productores (EDULEARN, INTED e ICERI) que son los que aglutinan el mayor número de publicaciones.

\section{Conclusiones}

Con el presente trabajo se ha podido constatar que la producción científica sobre el uso de las redes sociales como herramienta formativa en el periodo 2009-2018, está en continua expansión y focalizada en los países anglosajones y con presencia en revistas de reconocido prestigio y las bases de datos más importantes en el ámbito científico. A partir del estudio realizado y en función de los hallazgos obtenidos, se presentan las siguientes conclusiones:

- El período de mayor producción científica tuvo lugar entre 2015-2018 alcanzando la mayor productividad en el 2018 con 54 artículos. Por tanto como indicaban Oviedo, Muñoz \& Castellanos (2015) el campo de las redes sociales está en en expansión y aumentará su producción científica en diversos ámbitos.

- Un mayor número de citas no está asociado a un número elevado del índice de inmediatez debido a que depende de otro factor que es el número de artículos publicados.

- El tópico de estudio es muy dinámico ya que presenta una vida media de ocho años por tanto se concluye que desde el año 2018 se está produciendo una renovación en la literatura científica sobre redes sociales.

- Los papers en revistas constituyen más del 50\% de los documentos de la muestra lo que nos indica que estamos ante un campo que tiene trabajos de reconocido prestigio.

- El área y categoría más utilizada en la Web of Science es Education Educational Research, mientras que Communication, Education Scientific Disciplines y Computer Science tienen un lugar destacado. De esto se concluye por un lado, lo que indicaba Araujo (2019) que las redes sociales tienden puentes de comunicación entre profesorado y alumnado y entre el propio alumnado y por otro, la gran versatilidad que han proporcionado las redes sociales como herramienta de formación en diversos ámbitos Educativo, Médico, Tecnológico sin perder su origen como herramienta de comunicación.

- Los países más productores presentan los índices de aislamiento más elevados. Ello se deriva de que las revistas que presentan una mayor calidad científica en el área están en estos países y por tanto no tienen interés en exportar sus investigaciones a revistas de otros lugares. Estas conclusiones van en la línea de las indicadas por Miyahira (2008) estableciéndose una 
serie de indicadores de calidad para evitar que las revistas no exporten el conocimiento.

- Los equipos de trabajo están formados por un número que oscila entre uno a tres autores. De ello extraemos la conclusión que existe poca financiación en el campo lo que provoca que exista poca colaboración entre autores.

- Más del 50\% de las publicaciones tanto en el SCI y el SCIE pertenecen a revistas del Q1 lo que nos hace concluir que este campo de estudio presenta trabajos de gran calidad. Esta conclusión sigue la línea indicada por Quispe (2008) que corrobora la necesidad de que se trabaje con los cuartiles elevados como elemento de calidad más que con el factor de impacto y sus multiples problemas.

- Las actas de congresos tienen una mayor representación en los libros debido a que existen tres congresos (EDULEARN, INTED e ICERI) que son los que aglutinan el mayor número de documentos mientras que gran parte de libros publicados libros pero que tienen poca calidad científica. La existencia de gran cantidad de congresos nos puede estar indicando de un campo dinámico y en continua expansión. Se evidencia esta apertura como exponen Fueyo, Braga \& Fano (2015) que aunque las redes sociales nacieron con otras finalidades, su aplicación se está extendiendo a otros campos y en concreto al contexto educativo.

\section{Referencias bibliográficas}

Ajjan, H. \& Hartshorne, R. (2008). Investigating faculty decision to adopt web 2.0 technologies: Theory and empirical tests. The Internet and Higher Education, 11(2), 7180. doi: 10.1016/j.iheduc.2008.05.002

Araujo, J. C. (2019). El componente social. Un indicador del trabajo colaborativo online. EDMETIC, Revista de Educación Mediática y TIC, 8(1), 171-200. doi: https://doi.org/10.21071/edmetic. v8i1.11104

Attal, D., Cowher, M., Al-Hamadani, M., Schoger, J., Staley, A., \& Landercasper, J. (2015). Twitter Social Media is a effective tool for Breast Cancer Patient Education and Suport: Patient-Reported Outcomes by Survey. Journal of Medical Internet Research, 17 (7), 188

Awidi, I. T., Paynter, M., y Vujosevic, T. (2019). Facebook group in the learning design of a higher education course: An analysis of factors influencing positive learning experience for students. Computers \& Education, 129, 106-121, doi: https://doi. org/10.1016/j.compedu.2018.10.018

Begovic, E. (2011). The Effects of Social Networks on the Student-Teacher Relationship. En Paper presented at the Twenty-Fourth Annual Ford Forum. Eckerd College, Florida, US.

Brenley, D. B., y Covey, J. (2018). Risky behavior via social media: the role of reasoned and social reactive pathways. Computers in Human Behaviour, 78, 183-191. doi: https://doi.org/10.1016/j. chb.2017.09.036

Burton, R., \& Kleber, R. (1960). The half-life of some scientific and technical literature. American Documentation, 11 (1), 18-22

Burbules, N. (2014). Los significados de "aprendizaje ubicuo". Education Policy Analysis Archives/Archivos Analíticos de Políticas Educativas, 22(1), 1-10. 
Cabada, R., Estrada, M., Sanchez, L., Sandoval, G., Velazquez, J., \& Barrientos, J. (2009). Modeling student's learning styles in web 2.0 learning systems. World Journal on Educational Technology, 1 (2), 78-88.

Camps, D. (2008). Limitaciones de los indicadores bibliométricos de la evaluación de la actividad biomédica. Colombia Médica, 39 (1), 74-79.

Castro, F \& Hernández, S. (2019). El impacto de las redes sociales en el aprendizaje y formación educacional de los jóvenes. Caso de intervención psicológica en el área educativa Revista de Estudios Clínicos e Investigación Psicológica, 9 (17), 72-82.

Eid, M., \& Al-Jabari, I. (2016). Social networking, knowledge sharing, and student learning: The case of University students. Computers \& Education, 99(1), 14-27

Ferreiro, L., \& Martín, C. (1997). Técnicas bibliométricas aplicadas a los estudios de usuarios. Revista General de Información y Documentación, 7(2), 41-68.

Fueyo, A., Braga, G., y Fano, S. (2015). Redes sociales y educación: el análisis sociopolítico como asignatura pendiente. Revista Interuniversitaria de Formación del Profesorado, 82 (29.1), 119-129.

García-Martín, J., \& García-Sánchez, J. (2013). Patterns of Web 2.0 tool use among young Spanish people. Computers and Education, 67(1), 105-120. Doi.http://dx.doi.org/10.1016/j.compedu.2013.03.003

García, A., Froment, F., Bohórquez, R., \& Vieira, S. (2017). Análisis bibliométrico de la interacción profesor-alumno a través de las redes sociales. Pixel Bit. Revista de Medios y Educación, 51 (1), 53-67. Doi: http://dx.doi.org/10.12795/pixelbit.2017.i51.04

Gruzd, A., Staves, K., \& Wilk, A. (2011). Tenure and promotion in the age of online social media. Proceedings of the American Society for Information Science and Technology, 48(1), 1-9. http://dx.doi.org/10.1002/meet.2011.14504801154.

Hager, P. (2012) Informal learning: everyday living. En The Routledge international handbook of learning (pp. 207-215). Oxon, Rouhagtledge

Heffner, A. (1981). Founded research multiple authorship and subauthorship collaboration in four discipline. Scientometrics, 3 (1), 5-12

Hemmi, A., Bayne, S., \& Land, R. (2009). The appropriation and repurposing of social technologies in higher education. Journal of Computer Assisted Learning, 25(1), 19-30. http://dx.doi.org/10.1111/j.1365-2729.2008.00306.x.

Joosten, T. (2012). Social media for educators: Strategies and best practices. Hoboken, NJ, USA: Jossey-Bass.

Latchem, C. (2018). Open and Distance Non-formal Education. In C. Latchem, Open and Distance Non-formal Education in Developing Countries, . Springer: Singapore.

Lin, H., \& Tasai, C. (2011). College student's conceptions of learning management: the difference between traditional (face-to-face) instruction and web-based learning environment. Learning, Media and Technology, 36 (4), 437-452

Lorenzo, G., Scagliarini, C. (2018). Revisión Bibliométrica sobre la realidad aumentada en Educación. Revista General de Información y Documentación, 28 (1), 45-60. https://doi.org/10.5209/RGID.60805

Miyahira, J. (2008). Criterios de calidad de las revistas científicas. Revista médica herediana, 19(1), 1-4.

Kaplan, A., \& Haenlein, M. (2010). Users of the world, unite! The challenges and opportunities of social media. Business Horizons, 53 (1), 59-68. http://dx.doi.org/10.10106/j.bushor.2009.09.003.

Ko, W. (2012). A study of the relationships among effective learning, professional competence and learning performance in culinary field. Journal of Hospitality, Leisure, Sport \& Tourism Education, 11(1), 12-20. 
Kwon, O. \& Yixing, W. (2010). An empirical study of the factors affecting social network service use. Computers in Human Behavior, 26 (2), 254-263. Doi: 10.1016/j.chb.2009.04.011

Lotka, A. (1926). The frequency distribution of scientific productivity. Journal of Washington Academy of Sciences, 16 (12), 317-323.

López-Piñero, J., \& Terrada, M. (1992). Los indicadores bibliométricos y la evaluación de la actividad médico-científica (III). Los indicadores de producción, circulación y dispersión, consumo de la información y repercusión. Medicina Clínica, 98 (1), 142148.

Martín, M., Hernández-Suarez, C., \& Mendoza-Lizcano, S. (2017). Ambientes de aprendizaje basados en herramientas web para el desarrollo de competencias TIC en la docencia. Revista Perspectivas, 2 (1), 97-104. https://doi.org/10.22463/25909215.1282

Malesky, L. A. \& Peters, C. (2012). Defining appropriate professional behaviour for faculty and university students on social networking websites. Higher Education, 63 (1), 135151. doi:10.1007/s10734-011-9451-x

Montero, I., \& León, O. (2007). A guide for naming research studies in Psychology. International Journal of Clinical and Health Psychology, 7(3), 847-862

O'Reilly, T. (2005). What is Web 2.0: Design patterns and business models for the next generation of software? www.oreillynet.com/pub/a/oreilly/tim/news/2005/09/30/whatis-web-20.html. [Consulta: 8/2/2019]

Oviedo, M., Muñoz, M. \& Castellanos, M. (2015). La expansión de las redes sociales. Un reto para la gestión de marketing. Contabilidad y Negocios: Revista del Departamento Académico de Ciencias Administrativas, 10 (20), 59-69.

Quispe, C. (2004). ¿Es el Factor de Impacto un buen indicador para medir la calidad de las revistas científicas?: análisis de algunos problemas generados por su uso. Infobib, 3, 1-4

Rosa, A., Huertas, J., \& Blanco, J. (1996). Metodología de la historia de la psicología. Madrid: Alianza Editorial.

Robbie, D. \& Zeeng, L. (2008). Engaging student social networks to motivate learning: Capturing, analysing and critiquing the visual image. The International Journal of Learning, 15 (3), 153-160.

Roblyer, M., McDaniel, M., Webb, M., Herman, J., \& Witty, J. (2010). Findings on Facebook in higher Education: A comparison of college faculty and students uses and perceptions of social networking sites. Internet and Higher Education, 13(3), 134-140

Rueda-Clausen, C., Villa-Roel, C., \& Rueda-Clausen, C. (2005). Indicadores bibliométricos: origen, aplicación, contradicción y nuevas propuestas. MedUNAB, 8 (1), 29-36.

Ruiz, A. (2019). Competencia digital y TICs en interpretación: «renovarse o morir. EDMETIC, Revista de Educación Mediática y TIC, 8 (1), 55-71. doi: https:// doi.org/10.21071/edmetic. v8i1.11062

Selwyn, N. (2009). Faceworking: exploring student's Education related use of Facebook. Learning Media and Technology, 34(2), 157-174.

Teclehaimanot, B. \& Hickman, T. (2009). Student-teacher interaction on Facebook: What students find appropriate. TechTrends, 55(3), 19-30. doi:10.1007/s11528011-0494-8

Vallejo-Ruiz, M. (2005). Estudio longitudinal de la producción española de tesis doctorales en educación matemática (1975-2002) (Tesis Doctoral). Universidad de Granada. Granada. España

Xu, L., Yan, X. \& Zhang, Z. (2019). Research on the Causes of the "Tik Tok” App Becoming Popular and the Existing Problems. Journal of Advanced Management Science, 7 (2), 59-63. 